\title{
On the Primary Reaction Pathways in the Photochemistry of Nitro-Polycyclic Aromatic Hydrocarbons
}

\author{
Carlos E Crespo-Hernández*, Aaron Vogt R and Briana Sealey \\ Department of Chemistry and Center for Chemical Dynamics, Case Western Reserve University, 10900 Euclid Avenue, Cleveland, Ohio 44106, USA
}

\begin{abstract}
The primary reaction pathways in the photochemistry of nitro-polycyclic aromatic hydrocarbons under specific laboratory conditions are briefly summarized. In addition, photochemical data is presented for 2-nitronaphthalene, 1-nitronaphthalene, and 2-methyl-1-nitronaphthalene in cyclohexane and acetonitrile solutions under aerobic and anaerobic conditions. It is shown that molecular oxygen significantly reduces the photodegradation quantum yield of 1-nitronaphthalene and 2-methyl-1-nitronaphthalene by $63 \%$ and $81 \%$, respectively, whereas 2-nitronaphthalene is photoinert in both solvents under aerobic and anaerobic conditions. In addition, it is proposed that recombination of the aryl and nitrogen (IV) dioxide geminate radical pair within the solvent cage or internal conversion of an initially formed intramolecular charge transfer state may play an important role in the fraction of excited molecules that returns to the ground state in 1-nitronaphthalene and 2-methyl-1-nitronaphthalene. Scavenging of radical species by molecular oxygen and the generation of singlet oxygen in high yield are proposed to contribute to the photochemistry of these nitro-naphthalene derivatives in solution.
\end{abstract}

Keywords: Photochemistry of nitro-polycyclic aromatic compounds; Radical pair recombination; Excited-state dynamics; Environmental photochemistry; Ultrafast spectroscopy; Computational chemistry

\section{Introduction}

Nitro-polycyclic aromatic hydrocarbons (NPAHs) constitute one of the most troubling classes of environmental pollutants. Concern about these compounds arises partly from their ubiquity: NPAHs are released to the environment directly from a variety of incomplete combustion processes [1] and are also formed in situ by atmospheric reactions of polycyclic aromatic hydrocarbons (PAHs) [2-5]. They have been identified as components of grilled food as well as in diesel, gasoline, and wood smoke emissions, and are also commonly found in aquatic systems and in sediments [5-9].

Despite the potential negative impact of NPAHs to human health, the emission of NPAHs to the environment continues, primarily from municipal incinerators [10], motor vehicles (particularly from diesel exhausts) [11,12], and industrial power plants [13] among other sources. These emissions are likely to rise with the increasing consumption of petroleum and coal particularly in developing countries. It is estimated that NPAH in ambient air, either as vapor or particle accounts for $~ 50 \%$ of the total direct mutagenicity [14]. Epidemiological studies show that exposure to diesel exhaust and urban air pollution is associated with an increased risk of lung cancer [9]. The increase in lung cancer risk that results from human exposure to particulate air pollution and the detection of NPAH compounds in lungs of non-smokers with lung cancer have led to continued interest in assessing their potential risk to humans and also in monitoring their concentrations and fate in the environment [15].

Photochemical transformation is thought to be one of the main routes of natural removal of NPAHs in the environment $[8,16-19]$. Importantly, NPAHs are frequently more toxic than their parent PAHs $[8,20]$, and photochemical degradation of a number of NPAHs leads, in turn, to photoproducts, some of which are more toxic than their parent compounds $[8,20,21]$. However, the light-induced transformation mechanisms of NPAHs are still under debate and knowledge of their fates in the environment is of current interest [22-39]. Fundamental gaps remain in our knowledge of the elementary steps that lead to product formation with regard to the competition among available reaction pathways, the intermediate species involved in the photochemical transformations, and the degradation rates as a function of compound structure, added co-solutes, and micro-environment [26,34,35,40-43]. Consequently, development of laboratory models enabling accurate and quantitative measurements of these photochemical properties is essential for modeling the concentration and persistence of NPAHs in the environment and to understand their overall contribution to air quality [44-46]. Moreover, laboratory studies can facilitate the development of quantitative structure-activity relationships (QSARs), which connects photophysical and photochemical data to the physicochemical and structural properties of NPAHs [20,22,23,30,32,38,39,47]. In recent years, QSARs have been successfully developed [19,48,49], relating the toxicity, carcinogenicity, and mutagenicity of NPAHs with their physicochemical and structural properties.

The present work summarizes the primary reaction pathways that have been identified in the photochemistry of NPAHs under specific laboratory conditions. In addition, photochemical results of 2-nitronaphthalene, 1-nitronaphthalene, and 2-methyl-1nitronaphthalene in cyclohexane and acetonitrile solutions are presented that provide new insights about the photochemical fate of these nitro-aromatic compounds in the environment.

\section{Materials and Methods}

\section{Chemicals}

Cyclohexane (99.9\%) was obtained from Fisher Scientific. Acetonitrile (99.6\%) was obtained from Acros. Both solvents were used as received. 2-Nitronaphthalene, 1-nitronaphthalene and 2-methyl1-nitronaphthalene were obtained from Sigma-Aldrich (99.7\%, 99\% and 99\%, respectively). 2-nitronaphthalene, 1-nitronaphthalene and 2-methyl-1-nitronaphthalene are moderately toxic compounds.

*Corresponding author: Carlos E Crespo-Hernandez, Frank Hovorka Assistan Professor, Department of Chemistry, Case Western Reserve University, Cleveland, Ohio, USA, Tel: 1-216-368-1911; E-mail: carlos.crespo@case.edu

Received June 29, 2013; Accepted July 12, 2013; Published July 17, 2013

Citation: Crespo-Hernández CE, VogtRA, Sealey B (2013) On the Primary Reaction Pathways in the Photochemistry of Nitro-Polycyclic Aromatic Hydrocarbons. Mod Chem appl 1: 105. doi:10.4172/2329-6798.1000106

Copyright: @ 2013 Crespo-Hernández CE, et al. This is an open-access article distributed under the terms of the Creative Commons Attribution License, which permits unrestricted use, distribution, and reproduction in any medium, provided the original author and source are credited. 
Proper safety precautions were taken at all times to limit health risks. Due to the presence of a minor impurity in the commercial sample of 2-nitronaphthalene [39], recrystallized samples of 2-nitronaphthalene from methanol were used in the experiments reported in this work.

\section{Steady-state measurements}

Steady-state absorbance measurements were performed using a Cary 100 UV/Vis spectrometer (Varian, Inc.). Photodegradation experiments were performed using a $150 \mathrm{~W}$ Xe lamp (Newport-Oriel, Apex Source Arc, source model 66453, lamp model 6255), as reported recently [39]. The wavelength range of $275-375 \mathrm{~nm}$ was selected for irradiation by using a FGUVS11S colored glass filter (Schott), except for the determination of photodegradation rates, where a Pyrex filter was also used to restrict the irradiation wavelengths to the 300 to 375 $\mathrm{nm}$ range. The polychromatic light was focused through a $450 \mathrm{~mm}$ lens placed at $5 \mathrm{~cm}$ from the front of the lamp source. The sample was placed at $41 \mathrm{~cm}$ from the front of the lens. The beam width at the sample was $0.95 \mathrm{~cm}$. Solutions were contained in a $1 \mathrm{~cm}$ quartz cuvette (Starna, Inc.) and stirred continuously with a magnetic stir bar (Starna, Inc.) to assure a homogenous irradiation of the solutions at all times.

For the determination of the polychromatic photodegradation quantum yields, the change in concentration of the nitronaphthalene derivatives as a function of irradiation time was obtained using a HPLC (Shimadzu LC-20AD) with an amide column (Ascentis RP-Amide, 5 $\mu \mathrm{m}, 25 \mathrm{~cm} \times 4.6 \mathrm{~mm}$ ). The HPLC was used in order to separate the parent compounds from the photoproducts. Calibration curves were obtained for each nitronaphthalene derivative. At least 5 data points were used for each of the calibration curves, giving area under the chromatographic fraction versus concentration, as described recently [39]. An isocratic elution was used with a solvent composition of $80 \%$ acetonitrile and $20 \%$ water. A photodiode array detector (Shimadzu SPD-M20A) was used to measure the absorbance of the eluting compounds. Potassium ferrioxalate was used as an actinometer to measure the lamp intensity in photons/s [50]. Photodegradation yields were measured using the method recently developed by Dodson et al. [51] under $\mathrm{O}_{2}$ - and airsaturated conditions.

\section{Results and Discussion}

\section{Brief summary of the primary photochemical pathways in NPAHs}

In 1966, Chapman et al. correlated the probability of photoreactivity of NPAHs with the out-of-plane arrangement of the nitro group relative to the plane of the aromatic moiety [47]. NPAH compounds with parallel nitro substituents were proposed to be more stable to light degradation than those with perpendicular nitro substituents. This correlation is known as the Chapman's orientation-photoreactivity relationship. In general, nitro groups peri to two hydrogen atoms are expected to be in a perpendicular orientation (Figure 1a), while nitro groups peri only to one hydrogen atom are expected to stay in a parallel orientation relative to the aromatic rings (Figure 1b) [48,52-54]. The preferred perpendicular orientation of the nitro group peri to two hydrogen atoms is due to steric forces acting between the hydrogen atoms on the aromatic rings and the oxygen atoms on the nitro group, which restrict the torsion angle of the nitro group to an out-of-plane conformation.

Chapman proposed that the first step after excitation of compounds with nitro groups that are perpendicular to the aromatic rings is rearrangement of the nitro group to a nitrite intermediate (Figure 2 (2)). It was further proposed that perpendicular orientations increased probability of overlap between the half-vacant, non-bonding orbital of the nitro group and the adjacent orbital of the aromatic ring in the excited state [47]. This orbital overlap leads to the formation of an oxaziridine-type transition state (Figure $2(1)$ ) that can collapse to the nitrite intermediate (Figure 2 (2)). A dissociation reaction then produces nitrogen (II) oxide (NO*) and aryloxy (ArO*) radicals (Figure 2 (3)). Oxidation products such as quinones and hydroxyl compounds are expected to be formed subsequently from secondary reactions of the aryloxy radical intermediate. In parallel orientations, orbital overlap between the $\pi$-orbitals of the aromatic ring and the half-vacant, non-bonding orbital of the nitro group is unfavorable in the excited state, precluding the formation of the oxaziridine-type transition state (Figure 2)

However, it is still unclear which excited state is responsible for the formation of the nitrite intermediate and of the $\mathrm{NO}$ and $\mathrm{ArO}$ radicals. Figure 3 summarizes the primary electronic-energy relaxation pathways that have been proposed in the photochemistry of NPAH compounds. A generic NPAH compound ( $\left.\mathrm{NO}_{2}-\mathrm{Ar}\right)$ is used as example. A direct dissociation-recombination mechanism from the excited singlet state was first proposed by Brown [55]. In this mechanism, the C-N bond is weakened in the excited singlet state leading to the formation of the aryl (Ar•) and nitrogen (IV) dioxide geminate radical pair. This radical pair was suggested to rearrange to a nitrite intermediate, which then forms the aryloxy radical (pathway $2 b$ in Figure 3). Subsequently, Chapman proposed that an upper $n \pi^{*}$ triplet state (pathway $2 \mathrm{a}$ in Figure 3 ) is another plausible candidate for the formation of nitrogen (II) oxide and the aryloxy radicals but did not provide spectroscopic evidence of its participation [47].

Spectroscopic studies, mainly by Hamanoue [56-60], and Testa and their co-workers $[61,62]$ were subsequently performed in an effort to detect the proposed excited states and reactive intermediate species participating in the photochemistry of NPAH compounds. Studies by Hamanoue et al. using 9-nitroanthracene and several meso-substituted nitroanthracene derivatives suggested that NPAH compounds decay through two simultaneous pathways after light absorption [29,5660]. Singlet-to-triplet intersystem crossing was proposed as one of the relaxation pathways (path 1 in Figure 3). A second pathway involves the formation of nitrogen (II) oxide and the aryloxy radicals through a photoinduced dissociation reaction of the NPAH compound from an intermediate triplet state (path 2a in Figure 3). Electron spin resonance and transient absorption studies have been used to detect the radical

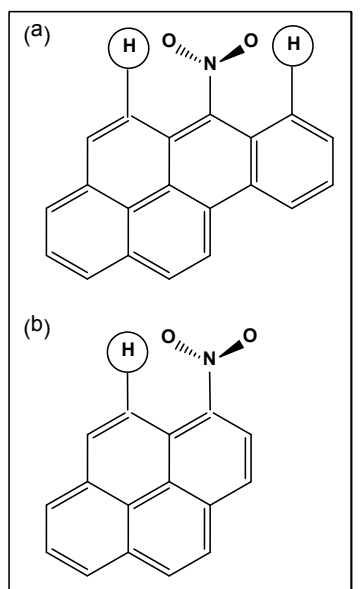

Figure 1: The nitro-group in (a) 6-nitrobenzo[a]pyrene is peri to two hydrogen atoms while in (b) 1-nitropyrene is peri to one hydrogen atom. 


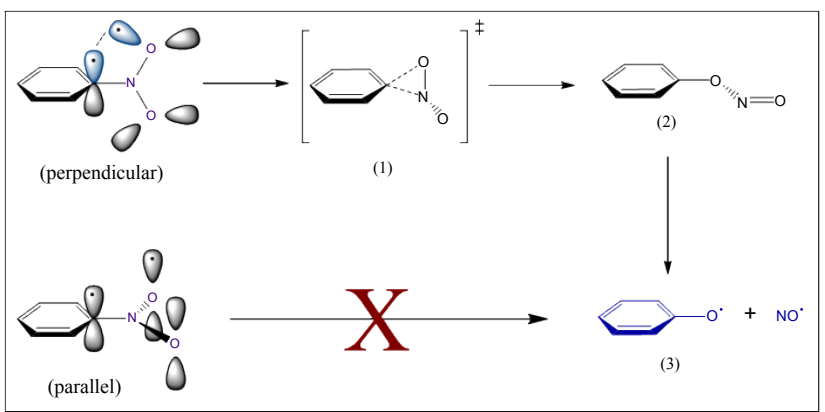

Figure 2: Schematic representation of the Chapman's orientation-photoreactivity relationship in the photochemistry of NPAH pollutants. Only one aromatic ring is shown for clarity [25]

intermediates formed from the dissociation reaction $[57,59,63]$. The lowest-energy excited triplet state has been detected using transient absorption, Raman spectroscopy, and steady state phosphorescence at low-temperature $[26,34,56,59,64,65]$. However, direct evidence of the population of the intermediate excited triplet state and of the excited state responsible for the formation of the nitrogen (II) oxide and the aryloxy radicals were not obtained because of the limited timeresolution and detection sensitivity used in those works. It has also been suggested that the lowest-energy excited triplet state in NPAH compounds may undergo intermolecular electron transfer or hydrogen abstraction depending on the solvent used (Figure 3) $[34,35,61,65,66]$, but direct spectroscopic evidence of these reactive intermediates is lacking (Figure 3).

Several groups have recently challenged Chapman's orientationreactivity relationship $[23,25,26,29,30]$. Warner et al. studied the photodegradation of several NPAH pollutants in solution and solid particles [23]. The authors grouped the NPAHs in three different categories based on their photoreactivity in solution. While several NPAH compounds followed Chapman's relationship others do not. It was concluded that the nitro group orientation is not the only factor controlling the photoreactivity of the NPAH pollutants [23], and recent works support this idea [25,29,32,34,38,39]. For instance, femtosecond broadband transient absorption spectroscopy in combination with computational methods has been recently used to study the excited state dynamics in 1-nitropyrene [25]. Ground state optimization of 1-nitropyrene results in a nitro group torsion angle of $32.8^{\circ}$ relative to the aromatic rings. Optimization of the lowestenergy excited singlet state resulted in a nitro group torsion angle of $0.07^{\circ}$. The transient absorption spectra revealed that the rotation of the nitro group in the excited singlet state occurs in $\sim 100$ femtoseconds [25]. Based on Chapman's relationship (Figure 2), the nitro to nitrite rearrangement should not be favored in the photochemistry of 1-nitropyrene. However, 1-hydroxypyrene is the major product in the photochemistry of 1-nitropyrene in nonpolar solvents and in the absence of oxygen $[8,26,34,35]$. The formation of 1-hydroxypyrene has been proposed to originate from the formation of the pyrenyloxyl radical, followed by hydrogen atom abstraction from the solvent [26]. This mechanism is inconsistent with Chapman's hypothesis if the nitro group of 1-nitropyrene relaxes to a planar conformation in the excited state. A dissociation-recombination mechanism was thus proposed as a potential precursor of 1-hydroxypyrene (pathway $2 \mathrm{~b}$ in Figure 3) [25]. However, direct evidence of the radical pair intermediate was not presented for 1-nitropyrene [25], but has been recently documented in 9-nitroanthracene [31]. Furthermore, experimental and computational data is now amassing in support of a direct photodissociation mechanism from an intramolecular charge transfer state as a competitive degradation channel in the photochemistry of NPAHs under anaerobic conditions $[25,29,31,35,38,39]$.

We have recently focused part of our effort toward investigating the electronic structure, steady-state UVA ( 320 to $400 \mathrm{~nm}$ ) photochemistry, and excited-state dynamics of 1-nitronaphtalene, 2-nitronaphthalene, and 2-methyl-1-nitronaphthalene in solution [29,38,39]. Quantumchemical calculations predict that the torsion angle in these nitroPAHs is modulated by electrostatic forces acting between the lone pairs of the oxygen atoms on the nitro group and the neighboring atoms on the aromatic moiety. The calculations also predict that there is a distribution of torsion angles in the ground state at room temperature for each NPAH. Upon excitation, the distribution of torsion angles accesses different regions of configurational space in the excited singlet state potential energy surface in each molecule, which is thought to modulate the time-resolved and steady-state photochemistry of these nitronaphthalene derivatives $[29,38,39]$. Furthermore, the calculations predict a branching of the Franck-Condon excited singlet state to populate two main relaxation pathways in these nitronaphthalene derivatives. The first, main decay channel connects the excited singlet state with a receiver, high-energy triplet state $\left(T_{n}\right)$ that has significant $\mathrm{n} \pi^{*}$ character. The second channel involves conformational relaxation, primarily of the nitro group, to populate an intramolecular charge transfer state in 1-nitronaphtalene and in 2-methyl-1-nitronaphthalene, whereas a ca. $5 \mathrm{kcal} / \mathrm{mol}$ energy barrier prohibits the population of the intramolecular change transfer state in 2-nitronaphthalene [38]. This energy barrier explains the lack of photoreactivity in 2-nitronaphthalene in the solvent used [39]. The dissociative channel is proposed to be responsible for the dissociation-recombination mechanism proposed originally by Brown [55], leading to the formation of the aryl radical (Ar•) and nitrogen (IV) dioxide geminate radical pair (Figure 3). This radical pair rearranges to a nitrite intermediate (2 in Figure 2), which then form the nitrogen (II) oxide (NO) and aryloxy (ArO) radicals ( 3 in Figure 2 and pathway $2 b$ in Figure 3). The formation of the NO and ArO radicals has been proposed to be the primary photochemical pathway leading to the observed photochemistry in 1-nitronaphthalene and 2-methyl-1-nitronaphthalene under anaerobic conditions [39].

\section{Role of aryl and nitrogen (IV) dioxide geminate radical pair recombination in the photochemical fate of nitronaphthalene derivatives}

An important aspect in the development of the kinetic model discussed above for the nitronaphthalene derivatives $[29,38,39]$ is the fact that internal conversion from the lowest-energy excited singlet state to the ground state is negligible, as time-resolved anisotropy measurements for 1-nitronaphthalene indicated [24]. This is further supported by recent CASPT2//CASSCF(12/12) calculations in which conical intersections between the $\mathrm{S}_{0}$ and excited singlet states were not found [37]. These nitronaphthalene derivatives also exhibit negligible fluorescence quantum yields of ca. $10^{-4}$ or less $[38,39]$. However, the

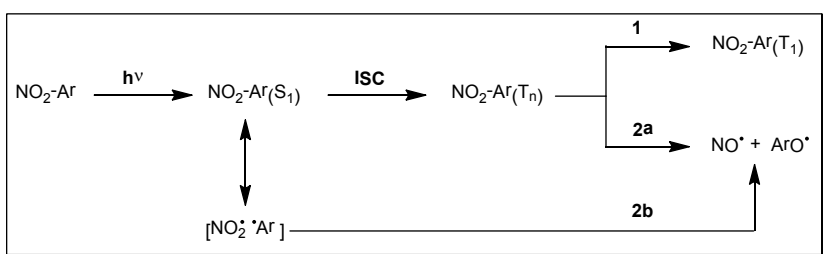

Figure 3: Summary of the proposed reaction pathways in the photochemistry of nitro-PAH compounds. 
reported photodegradation yield of 1-nitronaphthalene is ca. $10^{-3}$ and its triplet yield is only 64\% under anaerobic conditions [39], leaving a large fraction of the initial excited singlet state population unaccounted for. This result seems counterintuitive because it implies that close to $36 \%$ of the excited singlet state population in 1-nitronaphthalene should return back to the ground state. Similarly, the photodegradation yield of 2-nitronaphthalene is practically zero and the reported triplet yield is only $93 \%$ [39]. This implies that $7 \%$ of the excited singlet state population should decay back to the ground state. In the case of 2-methyl-1nitronaphthalene, the triplet yield is $33 \%$ and the photodegradation yield is $12 \%$ [39]. This leaves $55 \%$ of the excited-state population returning to the ground state unaccounted. A potential explanation is that the triplet quantum yields reported for these molecules might be underestimated by as much as $10-20 \%$, due to the energy transfer method used for their determination [39]. However, even under such an assumption, approximately $16 \%$ and $29 \%$ of the initial excited singlet state population returning to the ground state remain unaccounted for in 1-nitronaphthalene and 2-methyl-1-nitronaphthalene, respectively (Scheme 1).

To reconcile the fact that the time-resolved and the photochemical results available to date cannot account for the decay of all of the excited singlet state population, we propose that a large fraction of the aryl and nitrogen (IV) dioxide geminate radical pair decays back to the ground state by radical-pair recombination within the solvent cage, while only a small fraction dissociates to form the ArO and $\mathrm{NO}$ radicals (Scheme 1). An alternative explanation is that a fraction of the initially populated intramolecular charge transfer state internally converts back to the ground state before the geminate radical pair is formed. Either process can provide a reasonable explanation for the missing fraction of the excited singlet state population that returns back to the ground state. As explained above, a sizable energy barrier in the pathway leading to the intramolecular charge transfer state explains the triplet yield of close to unity in 2-nitronaphthalene [39], as well as its lack of photoreactivity of under anaerobic conditions [39]. The question arises as to whether this kinetic model can also explain the photoreactivity of the nitronaphthalene derivatives under aerobic conditions or if additional photochemical pathways are involved. For instance, the recently reported [39] high triplet yield in these nitronaphthalene derivatives may result in the efficient photosensitization of singlet oxygen, which can in turns lead to the formation of new oxidation products.

\section{Photodegradation rates and quantum yields in nitronaphthalene derivatives under anaerobic and aerobic conditions}

Figure 4 shows the changes in the absorption spectra of 1-nitronaphthalene as a function of irradiation time in the spectral

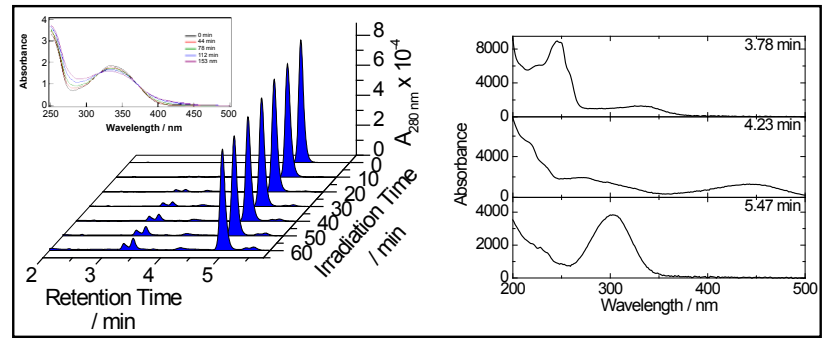

Figure 4: Photolysis of 1-nitronaphthalene using a Xe-lamp in cyclohexane solution and identification of the absorption spectra of the major products under air-saturated conditions.

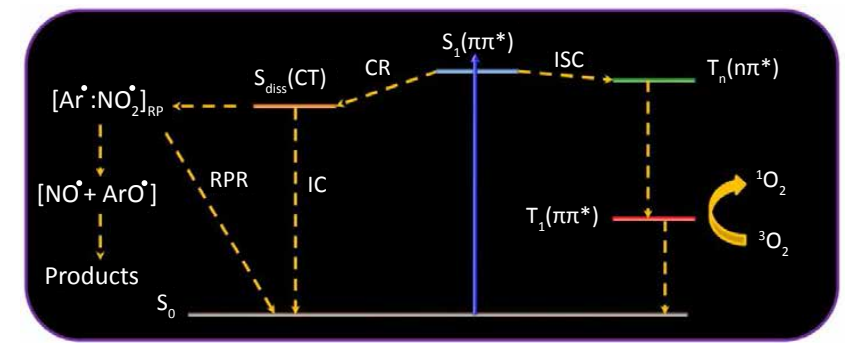

Scheme 1: Proposed reaction pathways in the photochemistry of 1-nitronaphthalene and 2-methyl-1-nitronaphthalene in cyclohexane and acetonitrile solutions. UVA excitation leads to an initial branching of the $\mathrm{S}_{1}$ state into two relaxation pathways: (1) ultrafast intersystem crossing (ISC) to the triplet manifold and (2) conformational relaxation (CR) of the nitro group to an intramolecular charge transfer state with dissociative character, $S$ (CT). The $\mathrm{S}_{\text {diss }}(\mathrm{CT})$ state population can dissociate to form the aryl (Ar) and nitrogen (IV) dioxide $\left(\mathrm{NO}_{2}{ }^{\circ}\right.$ ) geminate radical pair (RP) or decay non radiatively by internal conversion (IC) to the ground state. The geminate radical pair can either recombine within the solvent cage (RPR) or rearrange to form the aryloxy (ArO*) and nitrogen (II) oxide radical (NO), resulting in product formation. It is clear that molecular oxygen reduces the photodegradation yields significantly, likely forming singlet oxygen, whereas it is unclear whether or not the triplet state participate in the photochemistry of these nitronaphthalene derivatives [38]

region from 275 to $375 \mathrm{~nm}$ using a Xe-lamp as described in the methods section. These experiments were performed in cyclohexane under air-saturated conditions. The absorption spectra of the major products are also shown in Figure 4 under air-saturated conditions, using a photodiode array detector after HPLC separation of the reaction mixture. In particular, we highlight herein the absorption spectrum of the photoproduct with elution time of $4.23 \mathrm{~min}$. The absorption spectrum of this product shows a red-shifted absorption band with maximum at $\sim 445 \mathrm{~nm}$ relative to 1-nitronaphthalene. This is indicative of the formation of an oxidation product. This oxidation product might result from the aryloxy radical after hydrogen abstraction from the solvent or from a reaction of singlet oxygen with 1-nitronaphthalene. However, more work is needed to characterize the photoproducts of 1-nitronaphthalene and those resulting from the photolysis of 2-methyl-1-nitronaphthalene in different solvents and in the presence of different additives. While this is out of the scope of the present work, below we explore what can be learned regarding the primary photochemical pathways in these nitronaphthalene compounds from the determination of photodegradation rates and quantum yields in the presence or absence of molecular oxygen (Figure 4).

The photodegradation rates for 2-nitronaphthalene, 1-nitronaphthalene, and 2-methyl-1-nitronaphthalene were estimated from the changes in absorbance as a function of irradiation time for each nitronaphthalene derivative using absorption spectroscopy under air-saturated conditions. A Pyrex filter was used to block the radiation wavelengths below $300 \mathrm{~nm}$ (see the methods section for details). Precautions were taken to ensure that the integrated area of the initial absorption band above $300 \mathrm{~nm}$ (i.e., before the irradiation experiments began) were similar for the three nitronaphthalene derivatives. The changes in absorbance were converted to changes in concentration using the extinction coefficients reported in the literature for each compound [39]. A kinetic, zero-order rate equation was used to determine the photodegradation rates because the percent of degradation of each nitronaphthalene derivative was kept at $15 \%$ or less. In the case of 2-nitronaphthalene, no detectable changes in absorption spectrum were observed even after more than 6 hours of continuous irradiation in cyclohexane or acetonitrile solutions. Hence, it is assumed to be photoinert under the air- and $\mathrm{O}_{2}$-saturated conditions used in this 
work. Similar results were obtained recently for the photochemistry of 2-nitronaphthalene in acetonitrile under anaerobic conditions [39] (Figure 5).

Figure 5 shows the estimated photodegradation rates as a function of the calculate ground-state torsion angle for each compound. The torsion angles were obtained from density functional calculations in vacuum at the B3LYP/6-31++G(d,p) level of theory, as reported previously $[29,39]$. The photodegradation rates increase with an increase in the nitro-group torsion angle of the nitronaphthalene derivatives independent of the solvent used. This is in general agreement with Chapman's orientation-reactivity relationship (Figure 2) [47] and in good agreement with the photodegradation rates previously measured in the gas phase [22]. While the photodegradation rate of 1 -nitronaphthalene does not vary significantly in cyclohexane versus acetonitrile, the photodegradation rate of 2-methyl-1-nitronaphthalene is twofold higher in cyclohexane than in acetonitrile. The latter observation correlates with the smaller rate of intersystem crossing reported for this compound in cyclohexane $\left(2.7 \times 10^{12} \mathrm{~s}^{-1}\right)$ relative to acetonitrile $\left(4.8 \times 10^{12} \mathrm{~s}^{-1}\right)$ [38]. In other words, assuming that the solvent-dependent intersystem crossing rates equate to a decrease in the triplet yield of 2-methyl-1-nitronaphthalene in cyclohexane versus acetonitrile, an increase in the yield of the dissociative pathway would be expected (Scheme 1), which can lead to the observed increase in the photodegradation rate in cyclohexane. Another possibility is that there is a higher formation of singlet oxygen in cyclohexane than in acetonitrile for 2-methyl-1-nitronaphthalene [67], which leads to a significant increase in the formation of oxidative products. However, the participation of singlet oxygen in the formation of oxidation products seems to be insignificant. A more quantitative analysis of the potential participation of singlet oxygen in the photochemistry of 1-nitronaphthalene and 2-methyl-1-nitronaphthalene requires the determination of photodegradation yields in the presence and absence of molecular oxygen, which are reported next (Table 1).

Table 1 collects the photodegradation yields of 2-nitronaphthalene, 1-nitronaphthalene, and 2-methyl-1-nitronaphthalene in acetonitrile under $\mathrm{N}_{2}^{-}, \mathrm{O}_{2}^{-}$, and air-saturated conditions. The photodegradation quantum yields were obtained using a HPLC to separate the photoproducts from the parent compound, as explained in the method section. Analogous measurements in cyclohexane were impractical because the higher volatility of this solvent. As in the case of the photodegradation rate reported above, no photodegradation was observed in the irradiation experiments for 2-nitronaphthalene in the presence or absence of molecular oxygen and thus its

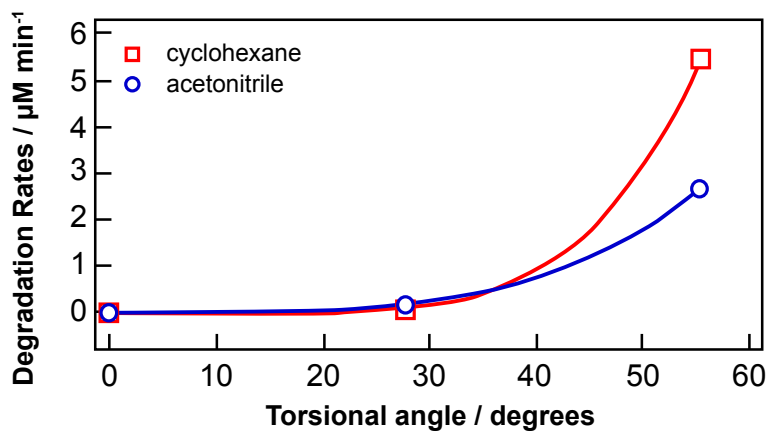

Figure 5: Correlation between the nitro-group orientation of the nitronaphthalene derivatives in the ground state with the photodegradation rates of 2-nitronaphthalene $\left(0.0^{\circ}\right)$, 1-nitronaphthalene $\left(27.7^{\circ}\right)$, and 2-methyl-1nitronaphthale $\left(55.4^{\circ}\right)$. Symbols are connected by a solid line to guide the eye.

\begin{tabular}{|l|l|l|l|}
\hline \multicolumn{1}{|c|}{ Molecule } & \multicolumn{1}{c|}{ Nitrogen [39] } & \multicolumn{1}{c|}{ Air } & \multicolumn{1}{c|}{ Oxygen } \\
\hline 2-nitronaphthalene & $\sim 0$ & $\sim 0$ & n.d. ${ }^{c}$ \\
\hline 1-nitronaphthalene & $0.0035 \pm 0.0004^{\mathrm{a}}$ & $0.0015 \pm 0.0004$ & $0.0013 \pm 0.0002$ \\
\hline 2-methyl-1-nitronaphthalene & $0.123^{\mathrm{b}} \pm 0.008$ & $0.043 \pm 0.002$ & $0.023 \pm 0.002$ \\
\hline
\end{tabular}

aerrors are equal to two standard deviations from three independent measurements ${ }^{b}$ value did not change significantly with freshly distilled acetonitrile $(0.126) ;{ }^{c}$ n.d. = not determined.

Table 1: Photodegradation yields of the nitronaphthalene derivatives in acetonitrile (initial solute concentration of ca. $150 \mu \mathrm{M}$ ).

photodegradation yield is reported as zero in Table 1. On the other hand, the photodegradation quantum yield of 1-nitronaphthalene and 2-methyl-1-nitronaphthalene decreases with an increase of molecular oxygen concentration. This might be indicative of quenching of the triplet state in the presence of molecular oxygen, leading to large yields of singlet oxygen generation in 1-nitronaphthalene [67] and 2-methyl1 -nitronaphthalene solutions. However, this will also suggest that singlet oxygen is unable to oxidize 1-nitronaphthalene or 2-methyl-1nitronaphthalene in acetonitrile, as has been observed in nitropyreno derivatives [68].

The suggestion above that singlet oxygen cannot oxidize the nitronaphthalene derivatives would also imply at first glance that the triplet state should play a large role in the photochemistry of 1-nitronaphthalene and 2-methyl-1-nitronaphthalene in acetonitrile, contrary to what has been recently proposed [39]. However, the reduction in the photodegradation yield under aerobic conditions can also be explained by assuming that molecular oxygen can scavenge one or more of the radicals formed in the intramolecular charge transfer pathway (Scheme 1). In fact, Garcia-Berrios and Arce have recently proposed that molecular oxygen can scavenge the pyrenyloxyl radical of 1-nitropyrene efficiently [35], thus reducing by $67 \%$ the formation yield of 1-hydroxypyrene, one of the major photoproducts [26]. If molecular oxygen can also scavenge one or more of the radicals formed in 1-nitronaphthalene and 2-methyl-1-nitronaphthalene, then this would explain part or all of the reduction in the photodegradation yield reported in Table 1 under aerobic conditions. For instance, recent experiments in aqueous solutions have shown that the radical anion of 1-nitronaphthalene can be scavenged by molecular oxygen [69]. Clearly, however, the experiments presented herein cannot prove or rule out the participation of the triplet state in the photoreactivity of these nitronaphthalene derivatives or the direct scavenging by molecular oxygen of one or more of the radical species proposed in Scheme 1. Altogether, it is clear that the photochemistry of these nitronaphthalene derivatives is more complex than previously thought and further steadstate and time-resolved photochemical investigations in the presence of triplet quenchers and radical/electron scavengers are warranted.

Experiments aimed at measuring and quantifying the generation of singlet oxygen in the photochemistry of 2-nitronaphthalene and 2-methyl-1-nitronaphthalene are essential to evaluate their potential phototoxicity, as has been recently shown for other NPAHs [20]. A high yield of singlet oxygen has been reported for 1-nitronaphthalene in both acetonitrile and cyclohexane solutions [67]. In particular, 2-nitronaphthalene might prove to be an excellent singlet oxygen generator upon UVA excitation, which can explain its high toxicity [19]. If so, 2-nitronaphthalene has the potential to be used in photosensitization and therapeutic applications, where the use of a photochemically-robust, photoinert sensitizer is required.

\section{Conclusions}

The major reaction pathways in the photochemistry of NPAHs under specific laboratory conditions have been presented. Readers interested 
in a broader discussion about the photochemistry of nitro-aromatic compounds are referred to previous monographs $[4,8,19,70,71]$. Despite recent progress at understanding the photodegradation mechanism of NPAHs, fundamental gaps remain in our knowledge of the elementary steps that lead to product formation. This is particularly evident regarding the competition among available reaction pathways, the intermediate species involved in the photochemical transformations, and the degradation rates as a function of compound structure, added co-solutes, and micro-environment.

New insights regarding the primary reaction pathways participating in the photochemistry of 2-nitronaphthalene, 1-nitronaphthalene, and 2-methyl-1-nitronaphthalene in cyclohexane and acetonitrile solutions under aerobic and anaerobic conditions were presented. It was shown that molecular oxygen significantly reduces the photodegradation quantum yield of 1-nitronaphthalene and 2-methyl-1-nitronaphthalene, whereas 2-nitronaphthalene is essentially photoinert. Recombination of the aryl and nitrogen (IV) dioxide geminate radical pair within the solvent cage was suggested to play an important role in the fraction of excited singlet state molecules that returns to the ground state in 1-nitronaphthalene and 2-methyl-1-nitronaphthalene. An alternative ground-state recovery pathway was proposed, where a fraction of the intramolecular charge transfer state internally converts to the ground state before the dissociation-recombination process occurs. Scavenging of radical species by molecular oxygen and the generation of singlet oxygen in high yield were proposed to contribute to the photochemistry of these nitro-naphthalene derivatives in solution. Singlet oxygen did not seem to oxidize the nitronaphthalene derivatives under the experimental conditions used in this work. The triplet state was suggested to play a minor role in the photochemistry of 1-nitronaphthalene and 2-methyl1-nitronaphthalene. Further studies are needed to corroborate or refute this latter hypothesis using triplet quenchers other than molecular oxygen.

\section{Acknowledgments}

This research was partially supported by the donors of the American Chemical Society Petroleum Research Fund. The authors also acknowledge the support to B.S. from the American Chemical Society and the Project SEED endowment.

\section{References}

1. World Health Organization (1989) Diesel and gasoline engine exhausts and some nitroarenes. International Agency for Research on Cancer 46: 41.

2. Atkinson R, Arey J (1994) Atmosphere chemistry of gas-phase polycyclic aromatic hydrocarbons: formation of atmospheric mutagens. Environ Health Perspect 102: 117-126.

3. Arey J, Zielinska B, Harger WP, Atkinson R, Winer AM (1988) The contribution of nitrofluoranthenes and nitropyrenes to the mutagenic activity of ambien particulate organic matter collected in southern California. Mutat Res 207: 4551.

4. Arey J, Atkinson R (2003) Photochemical reactions of PAHs in the atmosphere. PAHs: An ecotoxicological perspective. John Wiley \& Sons, Limited, UK.

5. Tokiwa H, Ohnishi $Y$ (1986) Mutagenicity and carcinogenicity of nitroarenes and their sources in the environment. Crit Rev Toxicol 17: 23-60

6. Möller $L$ (1994) In vivo metabolism and genotoxic effects of nitrated polycyclic aromatic hydrocarbons. Environ Health Perspect 102: 139-146.

7. Purohit V, Basu AK (2000) Mutagenicity of nitroaromatic compounds. Chem Res Toxicol 13: 673-692.

8. Yu H (2002) Environmental carcinogenic polycyclic aromatic hydrocarbons: photochemistry and phototoxicity. J Environ Sci Health C Environ Carcinog Ecotoxicol Rev 20: 149-183.

9. Arlt VM (2005) 3-nitrobenzanthrone, a potential human cancer hazard in diese exhaust and urban air pollution: a review of the evidence. Mutagenesis 20: $399-410$.
10. Kamiya A, Ose Y (1988) Isolation of dinitropyrene in emission gas from municipal incinerator and its formation by a photochemical reaction. Sci Total Environ 72: 1-9.

11. Henderson TR, Sun JD, Royer RE, Harvey TM, Hunt DH, et al. (1983) Triplequadrupole mass spectrometry studies of nitroaromatic emissions from different diesel engines. Environ Sci Technol 17: 443-449.

12. Handa T, Yamauchi T, Sawai K, Yamamura T, Koseki Y, et al. (1984) In situ emission levels of carcinogenic and mutagenic compounds from diesel and gasoline engine vehicles on an expressway. Environ Sci Technol 18: 895-902.

13. Hönor A, Arnold M, Hüsers N, Kleiböhmer W (1995) Monitoring polycyclic aromatic hydrocarbons in waste gases. Journal of Chromatography A 710 : 129-137.

14. Schauer C, Niessner R, Pöschl U (2004) Analysis of nitrated polycyclic aromatic hydrocarbons by liquid chromatography with fluorescence and mass spectrometry detection: air particulate matter, soot, and reaction product studies. Anal Bioanal Chem 378: 725-736.

15. Tokiwa H, Sera N, Horikawa K, Nakanishi Y, Shigematu N (1993) The presence of mutagens/carcinogens in the excised lung and analysis of lung cancer induction. Carcinogenesis 14: 1933-1938.

16. Kamens RM, Zhi-Hua F, Yao Y, Chen D, Chen S, et al. (1994) A methodology for modeling the formation and decay of nitro-PAH in the atmosphere. Chemosphere 28: 1623-1632.

17. Fan Z, Chen D, Birla P, Kamens RM (1995) Modeling of nitro-polycyclic aromatic hydrocarbon formation and decay in the atmosphere. Atmospheric Environment 29: 1171-1181.

18. Fan Z, Kamens RM, Hu J, Zhang J, McDow S (1996) Photostability of nitropolycyclic aromatic hydrocarbons on combustion soot particles in sunlight Environ Sci Technol 30: 1358-1364.

19. Janet K, Ulrich W, Inge M (2003) Selected nitro and nitro-oxy-polycyclic aromatic hydrocarbons. Environmental health criteria. World Health Organization, Geneva

20. Xia Q, Yin JJ, Zhao Y, Wu YS, Wang YQ, et al. (2013) UVA photoirradiation of nitro-polycyclic aromatic hydrocarbons-induction of reactive oxygen species and formation of lipid peroxides. Int J Environ Res Public Health 10: 1062-1084.

21. Victorin K (1994) Review of the genotoxicity of nitrogen oxides. Mutat Res 317 43-55.s

22. Phousongphouang PT, Arey J (2003) Rate constants for the photolysis of the nitronaphthalenes and methylnitronaphthalenes. Journal of Photochemistry and Photobiology A: Chemistry 157: 301-309.

23. Warner SD, Farant JP, Butler IS (2004) Photochemical degradation of selected nitropolycyclic aromatic hydrocarbons in solution and adsorbed to solid particles. Chemosphere 54: 1207-1215.

24. Morales-Cueto R, Esquivelzeta-Rabell M, Saucedo-Zugazagoitia J, Peon J (2007) Singlet excited-state dynamics of nitropolycyclic aromatic hydrocarbons direct measurements by femtosecond fluorescence up-conversion. J Phys Chem A 111: 552-557.

25. Crespo-Hernandez CE, Burdzinski G, Arce R (2008) Environmental photochemistry of nitro-PAHs: direct observation of ultrafast intersystem crossing in 1-nitropyrene. J Phys Chem A 112: 6313-6319.

26. Arce R, Pino EF, Valle C, Agreda J (2008) Photophysics and photochemistry of 1-nitropyrene. J Phys Chem A 121: 10294-10304.

27. Mohammed OF, Vauthey $E$ (2008) Excited-state dynamics of nitroperylene in solution: solvent and excitation wavelength dependence. J Phys Chem A 112 3823-3830.

28. Zugazagoitia JS, Collado-Fregoso E, Plaza-Medina EF, Peon J (2009) Relaxation in the triplet manifold of 1-nitronaphthalene observed by transient absorption spectroscopy. J Phys Chem A 113: 805-810.

29. Reichardt C, Vogt RA, Crespo-Hernández CE (2009) On the origin of ultrafast nonradiative transitions in nitro-polycyclic aromatic hydrocarbons: Excitedstate dynamics in 1-nitronaphthalene. J Chem Phys 131: 224518.

30. Vyas S, Onchoke KK, Rajesh CS, Hadad CM, Dutta PK (2009) Optical spectroscopic studies of mononitrated benzo[a]pyrenes. J Phys Chem A 113 12558-12565.

31. Plaza-Medina EF, Rodríguez-Córdoba W, Morales-Cueto R, Peon J (2011) 
Citation: Crespo-Hernández CE, Vogt RA, Sealey B (2013) On the Primary Reaction Pathways in the Photochemistry of Nitro-Polycyclic Aromatic Hydrocarbons. Mod Chem appl 1: 106. doi:10.4172/2329-6798.1000106

Page 7 of 7

Primary photochemistry of nitrated aromatic compounds: excited-state dynamics and NO- dissociation from 9-nitroanthracene. J Phys Chem A 115: 577-585

32. Plaza-Medina EF, Rodríguez-Córdoba W, Peon J (2011) Role of upper triplet states on the photophysics of nitrated polyaromatic compounds: $S(1)$ lifetimes of singly nitrated pyrenes. J Phys Chem A 115: 9782-9789.

33. Murudkar S, Mora AK, Singh PK, Nath S (2011) Origin of ultrafast excited state dynamics of 1-nitropyrene. J Phys Chem A 115: 10762-10766.

34. Arce R, Pino EF, Valle C, Negrón-Encarnación I, Morel M (2011) A comparative photophysical and photochemical study of nitropyrene isomers occurring in the environment. J Phys Chem A 115: 152-160.

35. García-Berríos ZI, Arce R (2012) Photodegradation mechanisms of 1-nitropyrene, an environmental pollutant: the effect of organic solvents, water, oxygen, phenols, and polycyclic aromatics on the destruction and product yields. J Phys Chem A 116: 3652-3664.

36. Healy RM, Chen Y, Kourtchev I, Kalberer M, O'Shea D, et al. (2012) Rapid formation of secondary organic aerosol from the photolysis of 1-nitronaphthalene: role of naphthoxy radical self-reaction. Environ Sci Technol 46: $11813-11820$

37. Orozco-Gonzalez Y, Coutinho K, Peon J, Canuto S (2012) Theoretical study of the absorption and nonradiative deactivation of 1-nitronaphthalene in the lowlying singlet and triplet excited states including methanol and ethanol solvent effects. J Chem Phys 137: 054307.

38. Vogt RA, Reichardt C, Crespo-Hernández CE (2013) Excited-state dynamics in nitro-naphthalene derivatives: Intersystem crossing to the triplet manifold in hundreds of femtoseconds. J Phys Chem A.

39. Vogt RA, Crespo-Hernández CE (2013) Conformational control in the population of the triplet state and photoreactivity of nitro-naphthalene derivatives.

40. Feilberg A, Kamens RM, Strommen MR, Nielsen T (1999) Modeling the formation, decay, and partitioning of semivolatile nitro-polycyclic aromatic hydrocarbons (nitronaphthalenes) in the atmosphere. Atmospheric Environment 33: $1231-1243$.

41. Feilberg A, Kamens RM, Strommen MR, Nielsen T (1999) Photochemistry and partitioning of semivolatile nitro-PAH in the atmosphere. Polycyclic Aromatic Compounds 14: 151-160.

42. Feilberg A, Nielsen T (2000) Effect of aerosol chemical composition on the photodegradation of nitro-polycyclic aromatic hydrocarbons. Environ Sci Technol 34: 789-797

43. Feilberg A, Nielsen T (2001) Photodegradation of nitro-PAHs in viscous organic media used as models of organic aerosols. Environ Sci Technol 35: 108-113.

44. Vione D, Maurino V, Minero C, Pelizzetti E, Harrison MA, et al. (2006) Photochemical reactions in the tropospheric aqueous phase and on particulate matter. Chem Soc Rev 35: 441-453.

45. Vione D, Minero C, Hamraoui A, Privat M (2007) Modelling photochemical reactions in atmospheric water droplets: an assessment of the importance of surface processes. Atmospheric Environment 41: 3303-3314.

46. Miet K, Le Menach K, Flaud PM, Budzinski H, Villenave E (2009) Heterogeneous reactions of ozone with pyrene, 1-hydroxypyrene, and 1-nitropyrene adsorbed on particles. Atmospheric Environment 43: 3699-3707.

47. Chapman OL, Heckert DC, Reasoner JW, Thackaberry SP (1966) Photochemical studies on 9-nitroanthracene. J Am Chem Soc 88: 5550-5554.

48. Fu PP, Herrero-Saenz D (1999) Nitro-polycyclic aromatic hydrocarbons: A class of genotoxic environmental pollutants. Journal of Environmental Science and Health, Part C: Environmental Carcinogenesis and Ecotoxicology Reviews 17: 1-43.

49. Vogt RA, Rahman S, Crespo-Hernandez CE (2010) Structure-activity relationships in nitro-aromatic compounds. Practical Aspects of Computational Chemistry 217-240.

50. Calvert JG, Pitts JN (1966) Photochemistry. Wiley: New York, USA.

51. Dodson LG, Vogt RA, Marks J, Reichardt C, Crespo-Hernández CE (2011) Photophysical and photochemical properties of the pharmaceutical compound salbutamol in aqueous solutions. Chemosphere 83: 1513-1523.

52. Fu PP, Chou MW, Beland FA (1988) Effects of nitro substitution on the in vitro metabolic activation of polycyclic aromatic hydrocarbons. In: Yang SK, Silverman BD (editions) polycyclic aromatic hydrocarbons carcinogenesis: structure-activity relationships. CRC Press, Boca Raton, USA.
53. Fu PP (1990) Metabolism of nitro-polycyclic aromatic hydrocarbons. Drug Metab Rev 22: 209-268.

54. Fu PP, Qui FY, Jung H, Von Tungeln LS, Zhan DJ, et al. (1997) Metabolism of isomeric nitrobenzo[a]pyrenes leading to DNA adducts and mutagenesis. Mutat Res 376: 43-51.

55. Brown HW, Pimentel GC (1958) Photolysis of nitromethane and methyl nitrite in an argon matrix; infrared detection of nitroxyl, HNO. J Chem Phys 29: 883-888.

56. Hamanoue K, Hirayama S, Nakayama T, Teranishi H (1980) Nonradiative relaxation process of the higher excited states of meso-substituted anthracenes. J Phys Chem 84: 2074-2078.

57. Hamanoue K, Amano M, Kimoto M, Kajiwara Y, Nakayama T, et al. (1984) Photochemical reactions of nitroanthracene derivatives in fluid solutions. $\mathrm{J} \mathrm{Am}$ Chem Soc 106: 5993-5997.

58. Hamanoue K, Nakayama T, Ushida K, Kajiwara K, Yamanaka S (1991) Photophysics and photochemistry of nitroanthracenes. Part 1-Primary processes in the photochemical reactions of 9-benzoyl-10-nitroanthracene and 9-cyano-10-nitroanthracene studied by steady-state photolysis and nanosecond laser photolysis. J Chem Soc Faraday Trans 87: 3365-3371.

59. Hamanoue K, Nakayama T, Kajiwara K, Yamanaka S (1992) Photophysics and photochemistry of nitroanthracenes. Part 2-Primary process in the photochemical reaction of 9-nitroanthracene studied by steady-state photolysis and laser photolysis. J Chem Soc Faraday Trans 88: 3145-3151.

60. Hamanoue K, Nakayama T, Amijima Y, Ibuki K (1997) A rapid decay channe of the lowest excited singlet state of 9-benzoyl-10-nitroanthracene generating 9-benzoyl-10-anthryloxy radical and nitrogen (II) oxide. Chemical Physics Letters 267: 165-170.

61. Hurley R, Testa AC (1968) Triplet-state yield of aromatic nitro compounds. J Am Chem Soc 90: 1949-1952.

62. TestaAC, Wild UP (1986) Holographic photochemical study of 9-nitroanthracene. J Phys Chem 90: 4302-4305.

63. Fukuhara K, Kurihara M, Miyata N (2001) Photochemical generation of nitric oxide from 6-nitrobenzo[a]pyrene. J Am Chem Soc 123: 8662-8666.

64. Anderson Jr RW, Hochstrasser RM, Lutz H, Scott GW (1974) Measurements of intersystem crossing kinetics using $3545 \AA$ picosecond pulses: nitronaphthalenes and benzophenone. Chemical Physics Letters 28: 153-157.

65. Fournier T, Tavender SM, Parker AW, Scholes GD, Phillips D (1997) Competitive energy and electron-transfer reactions of the triplet state of 1-nitronaphthalene: a laser flash photolysis and time-resolved Raman study. J Phys Chem A 101 : 5320-5326.

66. Görner H (2002) Photoreduction of nitronaphthalenes in benzene by N,Ndialkylanilines and triethylamine: a time-resolved UV-vis spectroscopic study. J Chem Soc Perkin Trans 2: 1778-1783.

67. Wilkinson F, McGarvey DJ, Olea AF (1994) Excited Triplet state interactions with molecular oxygen: Influence of charge transfer on the bimolecular quenching rate constants and the yields of singlet oxygen [0*2(1.delta.g)] for substituted naphthalenes in various solvents. J Phys Chem 98: 3762-3769.

68. Arce R personal communication.

69. Brigante M, Charbouillot T, Vione D, Mailhot G (2010) Photochemistry of 1-nitronaphthalene: a potential source of singlet oxygen and radical species in atmospheric waters. J Phys Chem A 114: 2830-2836.

70. Malkin J (1992) Photochemistry of Nitro Compounds. In: Photophysical and Photochemical Properties of Aromatic Compounds. CRC Press Inc., 426 pages.

71. Dopp D (1995) Photochemical Reactivity of the Nitro Group. In: CRC Handbook of Organic Photochemistry and Photobiology. CRC Press, Inc., Boca Raton, Florida, USA. 